\title{
Profiles of Coagulation and Fibrinolysis Activation-Associated Molecular Markers of Atypical Hemolytic Uremic Syndrome in the Acute Phase
}

\author{
Satoko Sakurai ${ }^{1}$, Hideki Kato ${ }^{1}$, Yoko Yoshida ${ }^{1}$, Yuka Sugawara ${ }^{1}$, Madoka Fujisawa ${ }^{1}$, Atsushi Yasumoto ${ }^{2}$, \\ Masanori Matsumoto ${ }^{3}$, Yoshihiro Fujimura ${ }^{4}$, Yutaka Yatomi ${ }^{2}$ and Masaomi Nangaku ${ }^{1}$ \\ ${ }^{1}$ Division of Nephrology and Endocrinology, the University of Tokyo Graduate School of Medicine, Tokyo, Japan \\ ${ }^{2}$ Department of Clinical Laboratory Medicine, the University of Tokyo Graduate School of Medicine, Tokyo, Japan \\ ${ }^{3}$ Department of Blood Transfusion Medicine, Nara Medical University, Nara, Japan \\ ${ }^{4}$ Japanese Red Cross Kinki Block Blood Center, Osaka, Japan
}

Aim: Atypical hemolytic uremic syndrome (aHUS), characterized by thrombotic microangiopathy (TMA), is a genetic, life-threatening disease which needs many differential diagnoses. This study aimed to reveal coagulation and fibrinolysis profiles in aHUS and secondary TMA patients. Furthermore, we investigated whether aHUS patients progress to, and meet, disseminated intravascular coagulation (DIC) criteria.

Methods: The acute phase samples were available in 15 aHUS and 20 secondary TMA patients. We measured PT-ratio, activated partial thromboplastin time (APTT), fibrinogen, fibrin degradation product (FDP), fibrin monomer complex (FMC), antithrombin (AT), plasmin- $\alpha 2$ plasmin inhibitor complex (PIC), and von Willebrand factor antigen (VWF:Ag). We examined and compared these tests among aHUS, secondary TMA patients, and healthy volunteer (HV), and evaluated whether patients with aHUS and secondary TMA met DIC criteria.

Results: PT-ratio, APTT, FDP, FMC and PIC in patients with aHUS and secondary TMA were higher than those in HV. Fibrinogen and AT showed no significant difference among three groups. VWF:Ag was higher in only aHUS patients. No tests showed significant difference between aHUS and secondary TMA patients. Three aHUS patients out of 15 met DIC criteria.

Conclusion: We revealed the profiles and distributions of coagulation and fibrinolysis tests of aHUS and secondary TMA patients. All tests were enhanced compared to HV; however, our results showed the no specificities in distinguishing aHUS from secondary TMA patients. We also clarified that some aHUS patients fulfilled DIC diagnostic criteria, indicating that DIC itself cannot be an exclusion criterion of aHUS.

\section{Key words: aHUS, TMA, DIC, Coagulation and fibrinolysis, Secondary TMA}

Abbreviations: atypical hemolytic uremic syndrome; aHUS, thrombotic microangiopathy; TMA, hemolytic uremic syndrome; HUS, Shiga toxin-producing Escherichia coli; STEC, hemolysis, complement factor H; $\mathrm{CFH}$, membrane cofactor protein; MCP or CD46, antithrombin; AT, fibrin monomer complex; FMC, plasmin- 22 plasmin inhibitor complex; PIC, disseminated intravascular coagulation; DIC, von Willebrand factor; VWF, healthy volunteer; HV, thrombotic thrombocytopenic purpura; TTP, heparin-induced thrombocytopenia; HIT, the Japanese Society on Thrombosis and Hemostasis; JSTH, fibrin degradation products; FDP, thrombin-antithrombin complex; TAT, soluble fibrin; SF, prothrombin fragment $1+2$; $F_{1+2}$, prothrombin time; PT, activated partial thromboplastin time; APTT, interquartile ranges; IQR, tissue factor; TF, total bilirubin; T-bil, lactate dehydrogenase; LDH, monoclonal gammopathy of undetermined significance; MGUS

\section{Introduction}

Atypical hemolytic uremic syndrome (aHUS) is one of the diseases belonging to thrombotic microangiopathy (TMA) ${ }^{1)}$, which is characterized by the triad of microangiopathic hemolytic anemia, thrombocytopenia, and acute kidney injury ${ }^{2}$. aHUS is a rare and complement-mediated disease. Patients with TMA are clinically diagnosed with aHUS after exclusion or evaluation of hemolytic uremic syndrome (HUS) due to 
Shiga toxin-producing Escherichia coli (STEC-HUS), thrombotic thrombocytopenic purpura (TTP), and secondary TMA resulting from drugs, malignancy, infection, transplantation, metabolism abnormality such as cobalamin deficiency, autoimmune diseases, malignant hypertension, pregnancy, or other causes ${ }^{3)}$.

aHUS is associated with congenital and acquired abnormalities of complement regulation, such as genetic variants, in complement factor $\mathrm{H}(\mathrm{CFH})^{4)}$, complement C3, complement factor B, complement factor I, membrane cofactor protein (MCP or CD46) ${ }^{5}$, or complement factor $\mathrm{H}$ autoantibodies ${ }^{6}$. However, the confirmation of genetic variants and measurement of antiCFH antibodies are still not available in clinical settings. Therefore, it is difficult to diagnose aHUS.

Although the crosstalk between complement activation and coagulation cascades has been described ${ }^{7-10)}$, few reports are available on the plasma levels of coagulation and fibrinolysis with aHUS patients in the acute phase ${ }^{11,12)}$. Thus, these tests in aHUS patients are not well known in clinical practice. We previously revealed that most aHUS patients in Japan showed increased fibrin degradation product (FDP) levels ${ }^{13)}$. This result suggests that coagulation and fibrinolysis might be perturbed in aHUS patients; however, detailed coagulation and fibrinolysis profiles have not been proven.

Furthermore, even though aHUS is diagnosed clinically, as mentioned above, and secondary TMA is also diagnosed when patients have underlying diseases that cause TMAs, the diagnosis is often difficult because similar symptoms, such as infection, are considered aHUS triggers and a cause of secondary TMA. Furthermore, reduced C3 levels have been found in only $50 \%$ of aHUS patients ${ }^{13,14)}$, and the genetic defects and/or complement activation have also been found in secondary TMA patients ${ }^{15}$. In addition, no biomarkers can distinguish aHUS and secondary TMA; thus, we comprehensively assess clinical symptoms. However, the usefulness of coagulation and fibrinolysis tests for differential diagnosis is unclear ${ }^{13)}$.

In addition, we have to differentiate TMA from similar conditions, especially disseminated intravascular coagulation (DIC) ${ }^{16-18)}$. DIC is usually one of the exclusion criteria to diagnose TMA; however, DIC is difficult to differentiate from TMA in clinical practice. It is unknown whether coagulation and fibrinolysis tests, especially in aHUS, are aggravated to fulfill the diagnosis of DIC.

\section{Aim}

This study's purpose was to reveal the detailed coagulation and fibrinolysis profiles in aHUS patients. Also, we evaluate the usefulness of coagulation and fibrinolysis to differentiate between aHUS and secondary TMA. Moreover, we clarified whether both aHUS patients and secondary TMA patients fulfilled DIC criteria for DIC.

\section{Methods}

\section{Subjects}

Patients were recruited from the aHUS cohort of Japan, established in Nara Medical University and the University of Tokyo Hospital from 2014 to $2017^{13}$. We excluded STEC-HUS, TTP, heparin-induced thrombocytopenia (HIT). Pernicious anemia, and apparent DIC due to malignancy, infection and hematological disorders based on consultation letters from physicians. Diagnoses of aHUS and secondary TMA were based on clinical guides for aHUS in Japan that were proposed by the Joint Committee of the Japanese Society of Nephrology and the Japan Pediatric Society in $2015^{3)}$. In brief, aHUS was defined as complementmediated HUS, and secondary TMA was defined as TMA arising from underlying diseases ${ }^{3)}$. Seventy-seven and 80 patients have been diagnosed as aHUS and secondary TMA, respectively.

Our aim is to investigate coagulation and fibrinolysis profiles by blood samples in the acute phase; therefore, we excluded patient samples collected after treatment, such as eculizumab administration, plasma therapy, or renal replacement therapy due to acute kidney failure. Moreover, we also ruled out the plasma samples with volumes less than or equal to $0.5 \mathrm{ml}$, and the plasma samples with residual platelet counts higher than $10,000 / \mu \mathrm{L}$ as inappropriate samples ${ }^{19)}$.

Finally, in this study, we retrospectively analyzed 15 patients with aHUS and 20 with secondary TMA. We examined the coagulation and fibrinolysis tests with these blood samples. Except for coagulation and fibrinolysis tests, laboratory data were retrospectively collected from consultation letters or questionnaires (Table 1). We recruited 15 healthy volunteers (HV; 7 men, 8 women, age ranging from 27 to 44 years old, median age 31 ) who had no physical signs and symptoms of disease and received no medications.

Address for correspondence: Masaomi Nangaku, Division of Nephrology and Endocrinology, The University of Tokyo Graduate School of Medicine, 7-3-1, Hongo, Bunkyo-ku, Tokyo, 113-8655, Japan E-mail: mnangaku-tky@umin.ac.jp

Received: March 2, 2019 Accepted for publication: July 22, 2019

Copyright@2020 Japan Atherosclerosis Society

This article is distributed under the terms of the latest version of CC BY-NC-SA defined by the Creative Commons Attribution License. 
Blood Collection and Assessment of Coagulation Factors

Citrated plasma samples were stored at $-80^{\circ} \mathrm{C}$ until use. The Automated Blood Coagulation Analyzer CS-2500 (sysmex, Kobe, Japan) was used for all the coagulation and fibrinolysis tests. Prothrombin time (PT) was measured using the Thromborel $S$ reagent (Siemens Healthcare Diagnostics, Marburg, Germany). The PT-ratio was calculated as the sample reaction time divided by normal plasma reaction time. Activated partial thromboplastin time (APTT) was measured using the Thrombocheck APTT-SLA (sysmex). Fibrinogen level was measured using the Thrombocheck Fib (L) (sysmex). Antithrombin III (AT) level was measured using the Berchrom Antitrombina III reagent (Siemens Healthcare Diagnostics). FDP level was measured using the Lias Auto P-FDP (sysmex). Fibrin monomer complex (FMC) level was measured using the Auto LIA ${ }^{\circledR}$ FM (Roche Diagnostics K.K., Tokyo, Japan). Plasmin- $\alpha 2$ plasmin inhibitor complex (PIC) level was measured using the Lias Auto PIC (sysmex). von Willebrand factor antigen (VWF:Ag) level was measured using the latex agglutination test (Siemens Healthcare Diagnostics). The value range of PT-ratio, FDP, fibrinogen, AT and FMC were defined by DIC of JSTH diagnostic criteria ${ }^{20)}$. We defined the largest values among the $\mathrm{HV}$ as the upper limit of APTT, PIC and VWF:Ag. ADAMTS13 activity were retrospectively collected from consultation letters or questionnaires.

\section{DIC Score}

DIC scores were given based on diagnostic criteria proposed by the Japanese Society on Thrombosis and Hemostasis (JSTH) 2017 ${ }^{20)}$ due to selective use of diagnostic criteria based on the underlying disease, application of molecular markers and antithrombin, and measures to reduce misdiagnoses. The patients were classified into three types: "hematopoietic disorder type", "infectious type", and "basic type", except for obstetric or newborn DIC. Patients having hematopoietic disorders were defined as the "hematopoietic type", and patients who had no hematopoietic disorder but had infections were defined as "infectious type". If the patients had no hematopoietic disorder and no infection, they were categorized as the "basic type". When an underlying disease could not be specified or there are many, the criteria for the "basic type" were applied unless the patients had hematopoietic disorders or infections.

For the basic type, scoring should be performed using the data for the platelet count, FDP level, fibrinogen level, PT-ratio, AT level, and coagulation activation-associated molecular markers. Zero to three points were given based on laboratory data. The total score was calculated, and a DIC diagnosis were established for the basic type, when the total was 6 points or more, for the infectious type, when the total was 5 points or more, and for the hematopoietic disorder type when the total was 4 points or more ${ }^{20)}$. Platelet counts were retrospectively collected from consultation letters or questionnaires. We substituted FMC level for soluble fibrin $(\mathrm{SF})^{21)}$ as coagulation activation-associated molecular markers.

\section{Statistical Analysis}

The data were expressed as the means \pm standard deviations for data showing a normal distribution and as medians and interquartile ranges (IQR) for data not showing a normal distribution. We used Student's $t$-test or Mann-Whitney $U$ test to compare the differences between aHUS and secondary TMA patients. We used Kruskal-Wallis test among three groups. If a difference was significant, all three pairs of groups were assessed individually with a two-sided Wilcoxon rank sum test. We considered $p$ values $<0.05$ as statistically significant. All analyses were carried out using JMP Pro 13.0 (SAS Institute, NC, USA).

\section{Ethics Statement}

This study was approved by the ethical committee of the University of Tokyo Hospital (IRB G10029) and registered to UMIN-CTR (UMIN000014869). It was performed in accordance with the 1964 Declaration of Helsinki and its later amendments, and the Ethical Guidelines on Clinical Studies of the Ministry of Health, Labor and Welfare of Japan. Written informed consent was obtained from all patients or their parents.

\section{Results}

\section{Patients Characteristics}

All the patients suspected of having aHUS underwent genetic screenings of C3, CFH, CBF, CFI, MCP, and DGKE, and auto-antibody screening against anti$\mathrm{CFH}$ abnormalities. Seven patients with aHUS were classified into three types according to the identified genetic abnormalities: C3 $(n=4)$, CFH $(n=1)$, MCP $(n=1)$. The one patient in this study with anti-CFH positive had no pathogenic genetics. The aHUS causes were unidentified in eight patients.

Secondary TMA patients were classified as follows: infection $(n=6)$, post renal transplantation $(n=2)$, post hematopoietic transplantation $(n=1)$, autoimmune diseases $(n=2)$, malignancy $(n=2)$, cytotoxic drugs $(n=1)$, malignant hypertension $(n=1)$, post operation $(n=1)$ and unknown $(n=4)$. The four unknown 
Table 1. Characteristics of patients

\begin{tabular}{lccc}
\hline & aHUS & secondary TMA & $P$ value \\
\hline Number & 15 & 20 & 0.001 \\
Age & $6(3-25)$ & $65.5(31-73.8)$ & 0.751 \\
Sex $(\mathrm{M}: \mathrm{F})$ & $4: 11$ & $10: 10$ & 0.689 \\
$\mathrm{Hb}(\mathrm{g} / \mathrm{dl})$ & $8.2(7.1-11.9)$ & $8(7.3-8.9)$ & 0.453 \\
$\mathrm{Ht}(\%)$ & $25(20.1-34.3)$ & $25.3(21.5-27.3)$ & 0.211 \\
Plt $\left(\times 10^{3} / \mu \mathrm{L}\right)$ & $31(27-74)$ & $52(29-87)$ & 0.006 \\
Albumin $(\mathrm{g} / \mathrm{dl})$ & $3.5 \pm 0.6$ & $3.2 \pm 0.6$ & 0.004 \\
T-Bil $(\mathrm{mg} / \mathrm{dl})$ & $2.3(1.4-3.2)$ & $608(0.4-1.1)$ & 0.185 \\
$\mathrm{LDH}(\mathrm{U} / \mathrm{l})$ & $1798(1281-2885)$ & $42.9 \pm 23.8$ & 0.258 \\
$\mathrm{BUN}(\mathrm{mg} / \mathrm{dl})$ & $55 \pm 24.1$ & $3.4(1.1-5.2)$ & 0.009 \\
$\mathrm{Cr}(\mathrm{mg} / \mathrm{dl})$ & $1.9(1.2-3.4)$ & $45(37.4-62.4)$ & \\
ADAMTS13: AC $(\%)$ & $71.4(66.3-86.5)$ & & \\
\hline
\end{tabular}

Data are expressed as the mean \pm SD or median (IQR)

Student's $t$-test was used for albumin and BUN to compare the differences between aHUS and secondary TMA patients. Mann-Whitney $U$ test was used for others.

The normal range for Plt: Child: $180-510 \times 10^{3} / \mu \mathrm{L}$, Adult: $158-348 \times 10^{3} / \mu \mathrm{L}$; T-Bil: Child: $0.3-0.9 \mathrm{mg} / \mathrm{dl}$, Adult: $0.4-1.5 \mathrm{mg} /$ dl; LDH: Child: 175-320 U/l, Adult: 124-222 U/l; Cr: Child: 0.25-0.48 mg/dl, Adult: M: 0.65-1.07, F: 0.46-0.79 mg/dl ${ }^{40,41)}$. aHUS; atypical hemolytic uremic syndrome, secondary TMA; secondary thrombotic microangiopathy, M; male, F; female, Hb; hemoglobin, Ht; hematocrit, Plt; platelet counts, T-bil; total bilirubin, LDH; lactate dehydrogenase, BUN; blood urea nitrogen, $\mathrm{Cr}$; creatinine, ADAMTS13; a disintegrin and metalloprotease with thrombospondin type 1 repeats 13 , AC: activity

cases did not have the typical demographics or family history of aHUS. Moreover, each of them had two underlying conditions. The first patient had monoclonal gammopathy of undetermined significance (MGUS) and Parkinson's disease, the second had MGUS and dyslipidemia, the third had hypertension and alcoholic liver disease, and the fourth had myelodysplastic syndrome and keratoacanthoma, none of which directly caused TMA. Taken together, we defined these four cases as secondary TMA with unknown cause.

The median age of aHUS patients was younger (median, 6; IQR, 3-25) than that of secondary TMA patients $(65.5 ; 31-73.8)(p<0.001)$. Plasma levels of total bilirubin [T-bil] (aHUS vs secondary TMA; median, 2.3 (IQR, 1.4-3.2) vs 0.5 (0.4-1.1)), lactate dehydrogenase [LDH] (1798 (1281-2885) vs 608 (389813)), ADAMTS13 activity (71.4 (66.3-86.5) vs 45 (37.4-62.4)) in aHUS patients were significantly higher than those in secondary TMA patients $(P<0.01$, respectively; Table 1).

Plasma Levels of Coagulation and Fibrinolysis Tests

We measured coagulation and fibrinolysis tests to aHUS and secondary TMA that are used to evaluate DIC.

Fig. 1 showed that PT-ratio $(P<0.001$, respectively) and APTT $(P<0.05$, respectively) in patients with aHUS and secondary TMA were significantly higher than HV. FDP level $(P<0.001$, respectively) was also significantly elevated in patients with aHUS and secondary TMA compared to HV. Fibrinogen level and AT level showed no significant differences among three groups, even though some patients with aHUS and secondary TMA had decreased fibrinogen levels less than $150 \mathrm{mg} / \mathrm{dL}$ (Fig. 1 and Table 2).

FMC level $(P<0.05$ and $P<0.001$, respectively) and PIC level $(P<0.001$, respectively) in patients with aHUS and secondary TMA were higher than those in HV (Fig. 1). Fig. 1 and Table 2 indicate that all aHUS patients $(n=15)$ showed that AT level was more than $70 \%$, PIC level was elevated, and FMC level was not higher than $12 \mu \mathrm{g} / \mathrm{ml}$ except for two patients who met DIC criteria.

VWF:Ag levels are not included in the DIC score evaluations; however, a previous study showed its level was elevated for aHUS patients compared to $\mathrm{HV}^{12)}$. VWF:Ag level $(P<0.001)$ in aHUS patients were significantly higher than that in $\mathrm{HV}$, while it showed no significant difference between aHUS and secondary TMA patients (Fig. 1). We revealed the profiles and abnormal distribution of coagulation and fibrinolysis tests in aHUS and secondary TMA patients; however, these tests were not specific to differentiate aHUS from secondary TMA.

\section{Evaluation of DIC Score}

We evaluated whether the patients with aHUS fulfilled the diagnostic criteria for DIC of JSTH. Three of fifteen patients with aHUS and five of twenty with secondary TMA met DIC criteria (Fig.2). All aHUS 

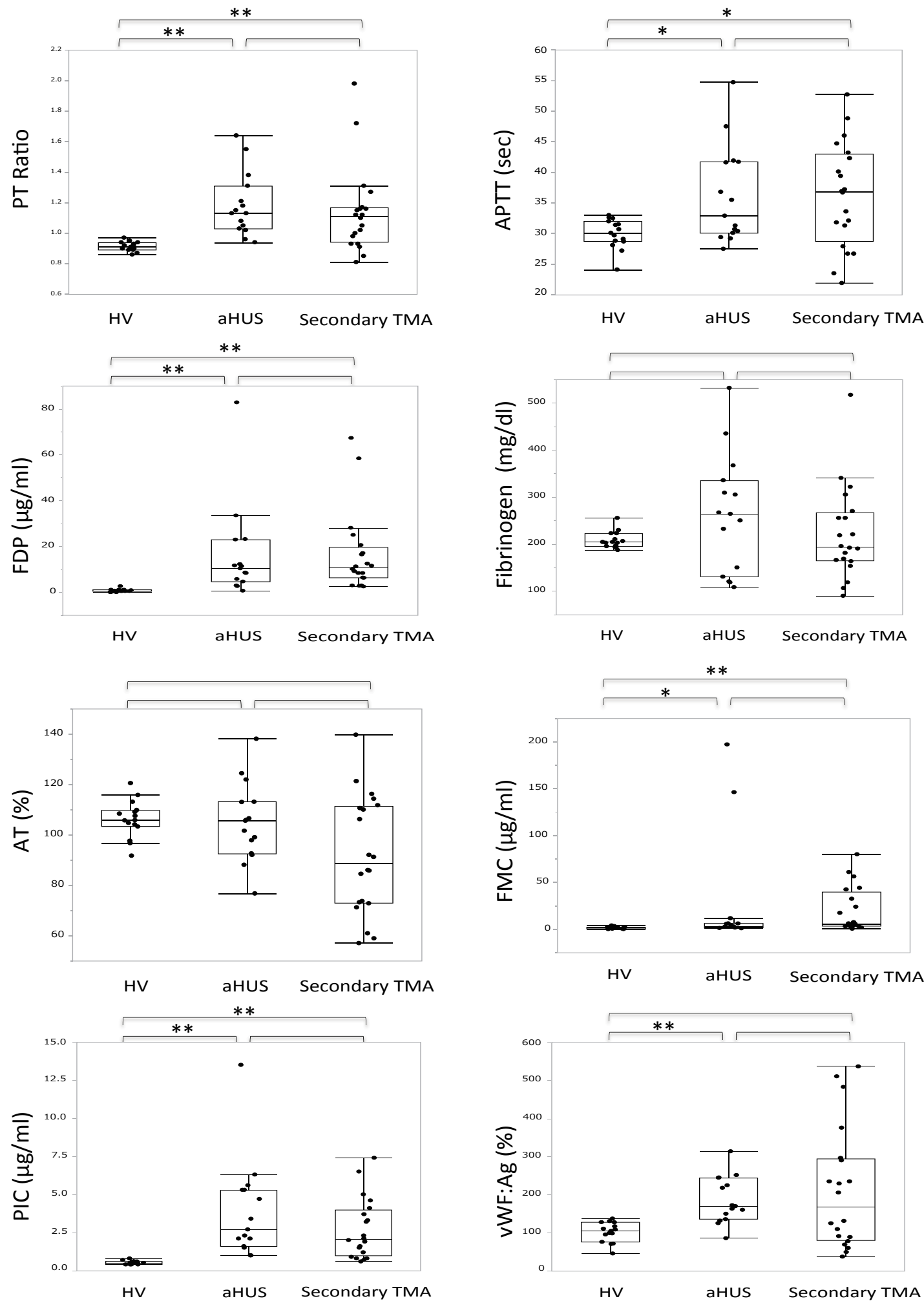

Fig. 1. The coagulation and fibrinolysis plasma levels in healthy volunteers, aHUS patients and secondary TMA patients. KruskalWallis test among three groups. If a difference was significant, all three pairs of groups were assessed individually by using a two-sided Wilcoxon rank sum test. ${ }^{* *} ;<0.001,{ }^{*} ;<0.05$

HV; healthy volunteer, aHUS; atypical hemolytic uremic syndrome, secondary TMA; secondary thrombotic microangiopathy, PT; prothrombin-time, FDP; fibrin degradation products, AT; antithombin, FMC; fibrin monomer complex, PIC; Plasmin- $\alpha 2$ plasmin inhibitor complex, VWF:Ag; von Willebrand factor antigen 
Table 2. Breakdown of values about aHUS and secondary TMA

\begin{tabular}{|c|c|c|c|c|}
\hline & Value ranges an & ints & aHUS n (\%) & secondary TMA n (\%) \\
\hline \multirow[t]{3}{*}{ PT-ratio } & $<1.25$ & $0 \mathrm{p}$ & $11(73)$ & $16(80)$ \\
\hline & $1.25 \leq-<1.67$ & $1 \mathrm{p}$ & $4(27)$ & $2(10)$ \\
\hline & $\geq 1.67$ & $2 \mathrm{p}$ & $0(0)$ & $2(10)$ \\
\hline \multirow[t]{2}{*}{ APTT (sec) } & $\leq 33$ & & $8(53)$ & $8(40)$ \\
\hline & $>33$ & & $7(47)$ & $12(60)$ \\
\hline \multirow[t]{4}{*}{ FDP $(\mu \mathrm{g} / \mathrm{mL})$} & $<10$ & $0 \mathrm{p}$ & $7(47)$ & $9(45)$ \\
\hline & $10 \leq-<20$ & $1 \mathrm{p}$ & $4(27)$ & $6(30)$ \\
\hline & $20 \leq-<40$ & $2 p$ & $3(20)$ & $3(15)$ \\
\hline & $\geq 40$ & $3 p$ & $1(6)$ & $2(10)$ \\
\hline \multirow[t]{3}{*}{ Fibrinogen $(\mathrm{mg} / \mathrm{dl})$} & $>150$ & $0 \mathrm{p}$ & $11(73)$ & $17(85)$ \\
\hline & $100 \leq-<150$ & $1 \mathrm{p}$ & $4(27)$ & $2(10)$ \\
\hline & $\leq 100$ & $2 p$ & $0(0)$ & $1(5)$ \\
\hline \multirow[t]{2}{*}{ AT (\%) } & $>70$ & $0 \mathrm{p}$ & $15(100)$ & $17(85)$ \\
\hline & $\leq 70$ & $1 \mathrm{p}$ & $0(0)$ & $3(15)$ \\
\hline \multirow[t]{2}{*}{ FMC (ug/ml) } & $\leq 12$ & $0 \mathrm{p}$ & $13(87)$ & $12(60)$ \\
\hline & $>12$ & $1 \mathrm{p}$ & $2(13)$ & $8(40)$ \\
\hline \multirow[t]{2}{*}{ PIC (ug/ml) } & $<0.8$ & & $0(0)$ & $2(10)$ \\
\hline & $\geq 0.8$ & & $15(100)$ & $18(90)$ \\
\hline \multirow[t]{2}{*}{ VWF:Ag (\%) } & $<138$ & & $4(27)$ & $10(50)$ \\
\hline & $\geq 138$ & & $11(73)$ & $10(50)$ \\
\hline
\end{tabular}

P; points, aHUS; atypical hemolytic uremic syndrome, secondary TMA; secondary thrombotic microangiopathy, n; number, AT; antithombin, FMC; fibrin monomer complex, PIC; Plasmin- $\alpha 2$ plasmin inhibitor complex, VWF:Ag; von Willebrand factor antigen

patients were classified into the basic type. As for secondary TMA, three patients were classified into the basic type, and two patients were classified into the infectious type (Fig. 2). Moreover, we also used DIC diagnostic criteria by the 1983 Japanese Ministry of Health and Welfare ${ }^{22)}$, and the same patients fulfilled previous DIC criteria.

Characteristics of three patients with aHUS who fulfilled DIC diagnostic criteria were as follows: they were all male, two patients were less than 1 year old, while one patient was 62 years old, one patient had genetic variant in $\mathrm{C} 3$, while the others had no predisposing genetic abnormalities.

\section{Discussion}

This study investigated the coagulation and fibrinolysis dynamics in aHUS patients. In the previous study, we found that aHUS patients had enhanced FDP levels while PT, APTT, and fibrinogen levels were variable ${ }^{13)}$. Therefore, further detailed analyses of coagulation and fibrinolysis in TMA patients were needed. We found that the PT-ratio, APTT, FDP levels, and
PIC levels were elevated in plasma; however, these markers are not likely to be useful for differential diagnosis of secondary TMA because the differences were not distinguished between aHUS and secondary TMA patients. Our results indicate that it is difficult to differentiate between aHUS and secondary TMA with underlying diseases using these markers. This may be because both complement abnormalities in aHUS and underlying diseases in secondary TMA perturb coagulation and fibrinolysis.

Our results showed that both plasma levels of T-bil and LDH in aHUS patients were significantly higher than those of secondary TMA. Even though the mean ages of the onset between aHUS and secondary TMA were significantly different due to the nature of the diseases and normal ranges of these plasma levels are slightly different based on age. The mean levels of T-bil and LDH in aHUS were much higher than the upper limits of their normal ranges for both adults and children. Though there have been no reports about T-bil level of aHUS and secondary TMA, previous reports show that medians of LDH level of aHUS ${ }^{13,23)}$ was higher than those of secondary TMA ${ }^{24,25)}$. Nota- 


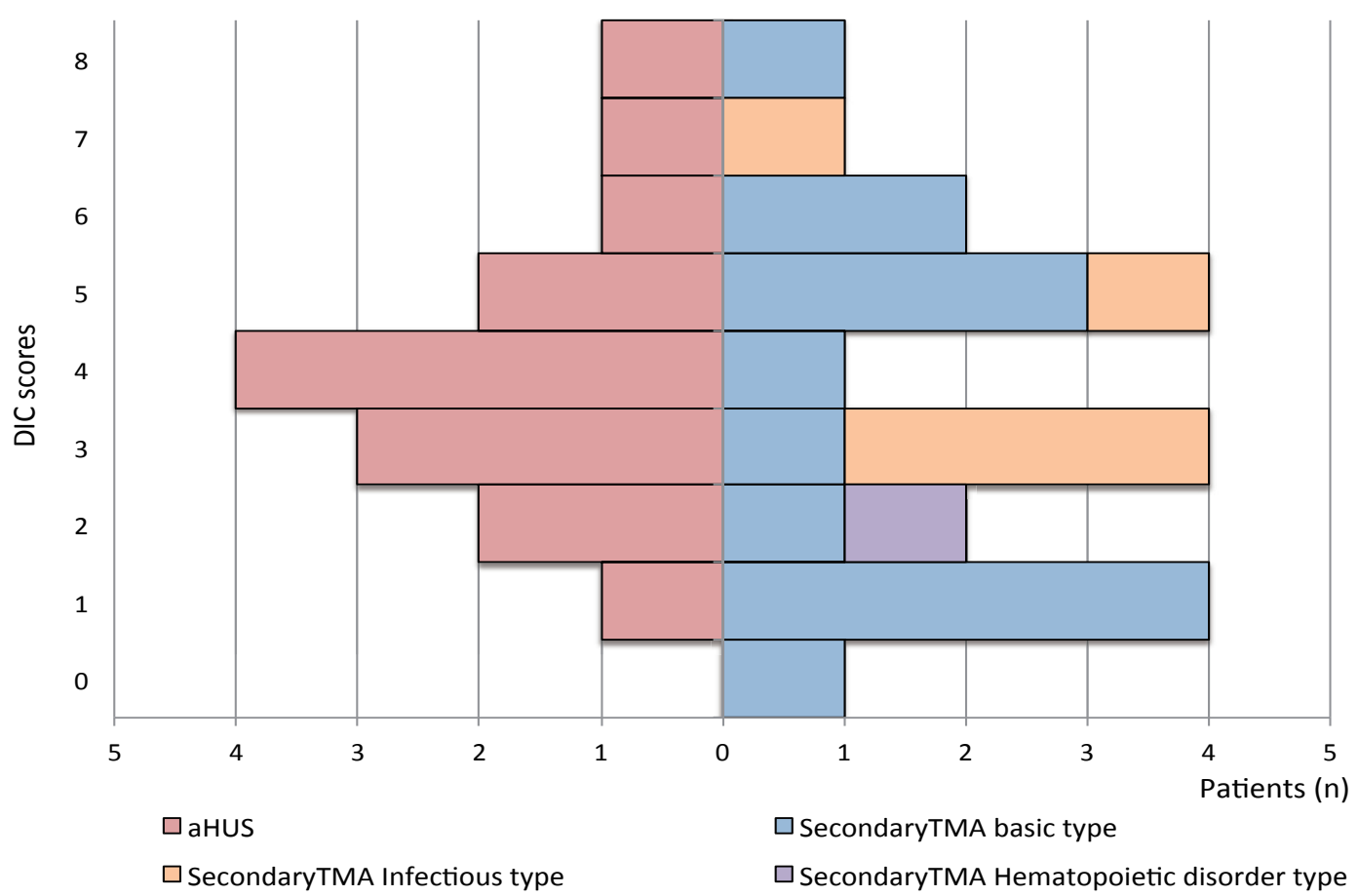

Fig. 2. Distribution of DIC scores in patients with aHUS and secondary TMA.

DIC scores were given based on the JSTH 2017 diagnostic criteria. All aHUS patients were classified into the basic type. Of the secondary TMA patients, fourteen patients were classified into the basic type, five patients were classified into the infectious type, and one patient were classified into the hematopoietic disorder type. Three of fifteen patients with aHUS and five of twenty with secondary TMA met DIC criteria. Red bar; basic type for aHUS patients, Blue bar; basic type for secondary TMA patients, Orange bar; infectious type for secondary TMA patients, Purple bar; hematopoietic disorder type for secondary TMA patients

JSTH; the Japanese Society on Thrombosis and Hemostasis, DIC; disseminated intravascular coagulation, aHUS; atypical hemolytic uremic syndrome, secondary TMA; secondary thrombotic microangiopathy

bly, these values ranged widely, suggesting that hemolysis with aHUS patients was more severe than secondary TMA.

VWF is released into the blood when vascular endothelial cell injury occurs ${ }^{26,27)}$. Our results showed that VWF:Ag levels in aHUS patients were significantly increased compared to HV; however, VWF:Ag levels in approximately half of secondary TMA patients were not elevated, and the difference between aHUS and secondary TMA was not statistically significant.

FMC is one of the coagulation activation-associated molecular markers, such as SF, thrombin-antithrombin complex (TAT), and prothrombin fragment $1+2\left(\mathrm{~F}_{1+2}\right)^{20)}$. These molecular markers are reportedly elevated in patients with aHUS ${ }^{11)}$ and DIC ${ }^{28-30)}$. Our result showed that FMC levels were markedly elevated in two of three patients with aHUS who fulfilled DIC criteria. Measuring FMC levels might be useful for detecting aHUS in patients who progress to DIC.

aHUS is caused by hyperactivation of the alternative complement pathway, and its effects on coagulation and fibrinolysis are not well understood today. The interaction between the complement system and the coagulation cascades has been reported ${ }^{7,8,31)}$. Complement activation releases anaphylatoxins, such as $\mathrm{C} 3 \mathrm{a}$ and $\mathrm{C} 5 \mathrm{a}$, and forms a membrane attack complex (C5b9). These complement activations also induce platelet activations and aggregation ${ }^{32-34)}$. Therefore, microvascular thrombosis, due to platelet activation, may activate coagulation and fibrinolysis. In addition, the complement activation can induce endothelial cell activation and expression of pro-coagulative tissue factor $(\mathrm{TF})^{8-10)}$. For examples, C5 ${ }^{35)}$ and the cytolytically inactive form of the C5b-9 ${ }^{36}$ can induce TF expression on human endothelial cells. These mechanisms might explain the activation of coagulation and fibrinolysis tests in acute aHUS patients.

Diagnosis of aHUS needs to exclude DIC, according to clinical guides for aHUS 2015 in Japan ${ }^{3)}$, and expert statements on the standard of care in critically ill adult patients with aHUS ${ }^{37)}$. However, patients with TMA and DIC can share similar clinical consequences, such as thrombocytopenia, microangiopathic hemolytic anemia, and microvascular thrombosis ${ }^{38)}$. A few articles reported aHUS patients fulfilled DIC diagnostic criteria ${ }^{12,39)}$. Our results also showed that some 
aHUS patients fulfilled new DIC diagnostic criteria, published in 2017, that adopted new coagulation and fibrinolysis markers ${ }^{20)}$. Therefore, our results suggested that aHUS might be hidden in excluded DIC. Moreover, our findings suggested that the distribution of DIC scores among clinically-diagnosed aHUS patients were unimodal, indicating aHUS patients sequentially progress to DIC. Further research on the relationship between coagulation and complement is required in the future.

There are several limitations in this study. First, the conditions of the blood samples were not constant because the samples of clinically-diagnosed aHUS patients were sent from various hospitals; thus, the blood sampling conditions and storage duration after sampling vary depending on the patient. Second, the patient numbers were small because aHUS is rare. Third, in this study, we examined coagulation and fibrinolysis tests only among clinically-diagnosed aHUS patients, and we used aHUS diagnostic criteria after excluding patients with apparent DIC due to malignancy, infection, and hematological disorders though there might be aHUS patients among apparent DIC patients. In the future, evaluation and diagnosis of aHUS among DIC patients might be necessary to detect aHUS in DIC patients.

\section{Conclusions}

We clarified that coagulation and fibrinolysis of aHUS patients was enhanced compared to HV, but showed the difficulties in distinguishing aHUS and secondary TMA by using these coagulation and fibrinolysis activation-associated molecular markers. Also, we showed that aHUS patients progress to fulfill DIC diagnostic criteria. Our results showed the importance of developing biological markers to detect aHUS, and further research is required on the interaction among complement, coagulation, and fibrinolysis.

\section{Acknowledgments}

We thank all the participants in this registry as well as the clinicians who enrolled patients in this study and contributed to the sample collection and clinical data acquisition.

\section{Funding}

This study was supported by research grants from the Ministry of Health, Labour, and Welfare of Japan (MN, HK); the Japan Society for the Promotion of Science, Grant-in-Aid for Scientific Research (C) (15K 09246, HK); the Japanese Association for Comple- ment Research (HK); the Practical Research Project for Rare/Intractable Diseases from Japan Agency for Medical Research and Development, AMED (17ek0109254 h0001, HK, MN, and YY).

\section{Competing Interests}

Satoko Sakurai: None

Hideki Kato: None.

Yoko Yoshida: None.

Yuka Sugawara: None.

Madoka Fujisawa: None.

Atsushi Yasumoto: None.

Masanori Matsumoto: MM received lecture fees from Asahi Kasei Pharma, Chugai Pharmaceutical, and Alexion Pharma; and research funds from Chugai Pharmaceutical and Asahi Kasei Pharma. However, this study has no association with that funding and the relationship did not affect the interpretation of this study's results.

Yoshihiro Fujimura: YF is a recipient of patent royalty for ADAMTS13 activity ELISA from Alfresa. However, this study has no association with that funding and the relationship did not affect the interpretation of this study's results.

Yutaka Yatomi: No competing interests to declare regarding the contents of this article.

Masaomi Nangaku: MN served as consultancy or advisory boards of Akebia, Bayer, Kyowa Hakko Kirin, Daiichi Sankyo, Astellas, Taisho, Mitsubishi Tanabe, JT, speaker bureaus of Kyowa Hakko Kirin, Daiichi Sankyo, MSD, Astellas, AstraZeneca, Alexion, Mitsubishi Tanabe, JT, and Chugai. However, this study has no association with that funding and the relationship did not affect the interpretation of this study's results.

\section{Human and Animal Rights}

Ethical approval: all procedures performed in studies involving human participants were in accordance with the ethical standards of the institutional and/or national research committee at which the studies were conducted (IRB approval number G10029) and with the 1964 Helsinki declaration and its later amendments or comparable ethical standards.

\section{Authors' Contributions}

SS and HK designed the study, collected and analyzed the data, and wrote the draft version of the manuscript. YY, YS and MF helped design the study and collect data and reviewed the manuscript. AY contributed to data analysis and experiment. MM, YF, YY and $\mathrm{MN}$ reviewed and finalized the manuscript for intel- 
lectual content. All authors read and approved the final manuscript.

\section{References}

1) George JN and Nester CM: Syndromes of thrombotic microangiopathy. N Engl J Med, 2014; 371: 654-666

2) Goodship TH, Cook HT, Fakhouri F, Fervenza FC, Fremeaux-Bacchi V, Kavanagh D, Nester CM, Noris M, Pickering MC, Rodriguez de Cordoba S, Roumenina LT, Sethi $S$ and Smith RJ: Atypical hemolytic uremic syndrome and C3 glomerulopathy: conclusions from a "Kidney Disease: Improving Global Outcomes" (KDIGO) Controversies Conference. Kidney Int, 2017; 91: 539-551

3) Kato H, Nangaku M, Hataya H, Sawai T, Ashida A, Fujimaru R, Hidaka Y, Kaname S, Maruyama S, Yasuda T, Yoshida Y, Ito S, Hattori M, Miyakawa Y, Fujimura Y, Okada $\mathrm{H}$ and Kagami S: Clinical guides for atypical hemolytic uremic syndrome in Japan. Clin Exp Nephrol, 2016; 20: $536-543$

4) Warwicker P, Goodship TH, Donne RL, Pirson Y, Nicholls A, Ward RM, Turnpenny P and Goodship JA: Genetic studies into inherited and sporadic hemolytic uremic syndrome. Kidney Int, 1998; 53: 836-844

5) Nester CM, Barbour T, de Cordoba SR, Dragon-Durey MA, Fremeaux-Bacchi V, Goodship TH, Kavanagh D, Noris M, Pickering M, Sanchez-Corral P, Skerka C, Zipfel P and Smith RJ: Atypical aHUS: State of the art. Mol Immunol, 2015; 67: 31-42

6) Dragon-Durey MA, Loirat C, Cloarec S, Macher MA, Blouin J, Nivet H, Weiss L, Fridman WH and FremeauxBacchi V: Anti-Factor $\mathrm{H}$ autoantibodies associated with atypical hemolytic uremic syndrome. J Am Soc Nephrol, 2005; 16: 555-563

7) Jokiranta TS: HUS and atypical HUS. Blood, 2017; 129: 2847-2856

8) Markiewski MM, Nilsson B, Ekdahl KN, Mollnes TE and Lambris JD: Complement and coagulation: strangers or partners in crime? Trends Immunol, 2007; 28: 184-192

9) Oikonomopoulou K, Ricklin D, Ward PA and Lambris JD: Interactions between coagulation and complement-their role in inflammation. Semin Immunopathol, 2012; 34: 151-165

10) Conway EM: Reincarnation of ancient links between coagulation and complement. J Thromb Haemost, 2015; 13 Suppl 1: S121-132

11) Cofiell R, Kukreja A, Bedard K, Yan Y, Mickle AP, Ogawa M, Bedrosian CL and Faas SJ: Eculizumab reduces complement activation, inflammation, endothelial damage, thrombosis, and renal injury markers in aHUS. Blood, 2015; 125: 3253-3262

12) Habe $K$, Wada $H$, Ito-Habe $N$, Hatada $T$, Matsumoto $T$, Ohishi K, Maruyama K, Imai H, Mizutani $\mathrm{H}$ and Nobori T: Plasma ADAMTS13, von Willebrand factor (VWF) and VWF propeptide profiles in patients with DIC and related diseases. Thromb Res, 2012; 129: 598-602

13) Fujisawa M, Kato H, Yoshida Y, Usui T, Takata M, Fujimoto M, Wada H, Uchida Y, Kokame K, Matsumoto M, Fujimura Y, Miyata T and Nangaku M: Clinical characteristics and genetic backgrounds of Japanese patients with atypical hemolytic uremic syndrome. Clin Exp Nephrol, 2018; 22: 1088-1099

14) Noris M, Caprioli J, Bresin E, Mossali C, Pianetti G, Gamba S, Daina E, Fenili C, Castelletti F, Sorosina A, Piras R, Donadelli R, Maranta R, van der Meer I, Conway EM, Zipfel PF, Goodship TH and Remuzzi G: Relative role of genetic complement abnormalities in sporadic and familial aHUS and their impact on clinical phenotype. Clin J Am Soc Nephrol, 2010; 5: 1844-1859

15) Yoshida Y, Kato H, Ikeda Y and Nangaku M: Pathogenesis of Atypical Hemolytic Uremic Syndrome. J Atheroscler Thromb, 2019; 26: 99-110

16) Taylor FB, Jr., Toh CH, Hoots WK, Wada H and Levi M: Towards definition, clinical and laboratory criteria, and a scoring system for disseminated intravascular coagulation. Thromb Haemost, 2001; 86: 1327-1330

17) Levi M, de Jonge E, van der Poll $\mathrm{T}$ and ten Cate H: Disseminated intravascular coagulation. Thromb Haemost, 1999; 82: 695-705

18) Laurence J, Haller H, Mannucci PM, Nangaku M, Praga $\mathrm{M}$ and Rodriguez de Cordoba S: Atypical hemolytic uremic syndrome (aHUS): essential aspects of an accurate diagnosis. Clin Adv Hematol Oncol, 2016; 14 Suppl 11: 2-15

19) Daves M, Giacomuzzi K, Tagnin E, Jani E, Adcock Funk DM, Favaloro EJ and Lippi G: Influence of centrifuge brake on residual platelet count and routine coagulation tests in citrated plasma. Blood Coagul Fibrinolysis, 2014; 25: 292-295

20) Asakura H, Takahashi H, Uchiyama T, Eguchi Y, Okamoto K, Kawasugi K, Madoiwa S and Wada H: Proposal for new diagnostic criteria for DIC from the Japanese Society on Thrombosis and Hemostasis. Thromb J, 2016; 14: 42

21) Suzuki A, Ebinuma H, Matsuo M, Miyazaki $O$ and Yago $\mathrm{H}$ : The monoclonal antibody that recognizes an epitope in the C-terminal region of the fibrinogen alpha-chain reacts with soluble fibrin and fibrin monomer generated by thrombin but not with those formed as plasmin degradation products. Thromb Res, 2007; 121: 377-385

22) Kobayashi N, Maekawa T, Takada M, Tanaka H and Gonmori H: Criteria for diagnosis of DIC based on the analysis of clinical and laboratory findings in 345 DIC patients collected by the Research Committee on DIC in Japan. Bibl Haematol, 1983; 265-275

23) Cataland SR, Holers VM, Geyer $S$, Yang $S$ and Wu HM: Biomarkers of terminal complement activation confirm the diagnosis of aHUS and differentiate aHUS from TTP. Blood, 2014; 123: 3733-3738

24) Zhang T, Chen H, Liang S, Chen D, Zheng C, Zeng C, Zhang $\mathrm{H}$ and Liu Z: A non-invasive laboratory panel as a diagnostic and prognostic biomarker for thrombotic microangiopathy: development and application in a Chinese cohort study. PLoS One, 2014; 9: e111992

25) Cavero T, Rabasco C, Lopez A, Roman E, Avila A, Sevillano A, Huerta A, Rojas-Rivera J, Fuentes C, Blasco M, Jarque A, Garcia A, Mendizabal S, Gavela E, Macia M, Quintana LF, Maria Romera A, Borrego J, Arjona E, Espinosa M, Portoles J, Gracia-Iguacel C, Gonzalez-Parra E, Aljama P, Morales E, Cao M, Rodriguez de Cordoba S and Praga M: Eculizumab in secondary atypical haemo- 
lytic uraemic syndrome. Nephrol Dial Transplant, 2017; 32: $466-474$

26) Moake JL, Turner NA, Stathopoulos NA, Nolasco LH and Hellums JD: Involvement of large plasma von Willebrand factor (vWF) multimers and unusually large vWF forms derived from endothelial cells in shear stress-induced platelet aggregation. J Clin Invest, 1986; 78: 1456-1461

27) Galbusera M, Benigni A, Paris S, Ruggenenti P, Zoja C, Rossi $C$ and Remuzzi G: Unrecognized pattern of von Willebrand factor abnormalities in hemolytic uremic syndrome and thrombotic thrombocytopenic purpura. J Am Soc Nephrol, 1999; 10: 1234-1241

28) Kushimoto S, Wada H, Kawasugi K, Okamoto K, Uchiyama T, Seki $Y$, Hatada T, Imai $\mathrm{H}$ and Nobori T: Increased ratio of soluble fibrin formation/thrombin generation in patients with DIC. Clin Appl Thromb Hemost, 2012; 18: 628-632

29) Wada H, Kobayashi T, Abe Y, Hatada T, Yamada N, Sudo A, Uchida A and Nobori T: Elevated levels of soluble fibrin or D-dimer indicate high risk of thrombosis. J Thromb Haemost, 2006; 4: 1253-1258

30) Okamoto K, Takaki A, Takeda S, Katoh H and Ohsato K: Coagulopathy in disseminated intravascular coagulation due to abdominal sepsis: determination of prothrombin fragment $1+2$ and other markers. Haemostasis, 1992; 22: 17-24

31) Amara U, Flierl MA, Rittirsch D, Klos A, Chen H, Acker B, Bruckner UB, Nilsson B, Gebhard F, Lambris JD and Huber-Lang M: Molecular intercommunication between the complement and coagulation systems. J Immunol, 2010; 185: 5628-5636

32) Martel C, Cointe S, Maurice P, Matar S, Ghitescu M, Theroux P and Bonnefoy A: Requirements for membrane attack complex formation and anaphylatoxins binding to collagen-activated platelets. PLoS One, 2011; 6: e18812
33) Polley MJ and Nachman RL: Human platelet activation by C3a and C3a des-arg. J Exp Med, 1983; 158: 603-615

34) Wiedmer T and Sims PJ: Effect of complement proteins C5b-9 on blood platelets. Evidence for reversible depolarization of membrane potential. J Biol Chem, 1985; 260: 8014-8019

35) Ikeda K, Nagasawa K, Horiuchi T, Tsuru T, Nishizaka H and Niho Y: C5a induces tissue factor activity on endothelial cells. Thromb Haemost, 1997; 77: 394-398

36) Tedesco F, Pausa M, Nardon E, Introna M, Mantovani A and Dobrina A: The cytolytically inactive terminal complement complex activates endothelial cells to express adhesion molecules and tissue factor procoagulant activity. J Exp Med, 1997; 185: 1619-1627

37) Azoulay E, Knoebl P, Garnacho-Montero J, Rusinova K, Galstian G, Eggimann P, Abroug F, Benoit D, von Bergwelt-Baildon M, Wendon J and Scully M: Expert Statements on the Standard of Care in Critically Ill Adult Patients With Atypical Hemolytic Uremic Syndrome. Chest, 2017; 152: 424-434

38) Kurosawa $S$ and Stearns-Kurosawa DJ: Complement, thrombotic microangiopathy and disseminated intravascular coagulation. J Intensive Care, 2014; 2: 65

39) Abe T, Sasaki A, Ueda T, Miyakawa Y and Ochiai H: Complement-mediated thrombotic microangiopathy secondary to sepsis-induced disseminated intravascular coagulation successfully treated with eculizumab: A case report. Medicine (Baltimore), 2017; 96: e6056

40) Department of Clinical Laboratory, The University of Tokyo Hospital [http://lab-tky.umin.jp/patient/ketueki.pdf] Accessed 7 June 2019

41) National Center for Child Health and Development [https://sogo-igaku.co.jp/lec_in_ped/0302.html] Accessed 7 June 2019 